\title{
Direct numerical simulation of Mhd heat transfer of the liquid metal in a horizontal pipe with the joint effect of the longitudinal magnetic field and thermo-gravitational convection
}

\author{
Akhmedagaev Ruslan ${ }^{1, *}$, and Listratov Yaroslav ${ }^{1}$ \\ ${ }^{1}$ National Research University «Moscow Power Engineering Institute», 111250 Moscow, Russia
}

\begin{abstract}
The results of the direct numerical simulation (DNS) of the MHD heat transfer in turbulent flow of liquid metal (LM) in a horizontal pipe under the joint influence of a longitudinal magnetic field (MF) and thermo-gravitational convection (TGC) are presented. Discusses the characteristics of hydrodynamics and heat transfer in the longitudinal MF in the absence of TGC. Suppression of turbulent transport is observed with an increase in the Hartmann number: the heat transfer coefficients, the friction coefficient. The authors calculated the effect of TGC in a strong longitudinal MF for a homogeneous heating. Investigated the averaged fields of velocity and temperature, heat transfer characteristics, the distribution of wall temperature along the perimeter of the cross section of the pipe. The effect of TGC on the velocity field is affected stronger than in the temperature field.
\end{abstract}

\section{Introduction}

Currently, the world community is working on the creation of an international experimental thermonuclear reactor (ITER), which is being built in Cadarache (France). Despite the water concept of cooling the reactor blanket, it is planned to design separate liquid metal modules for the production of tritium, obtained as a result of lithium irradiation by neutrons. In ITER liquid metal system will work if you have a very strong MF and the research problem of the regularities of hydrodynamics and heat transfer under such conditions at a current LM is very relevant [Ошибка! Источник ссылки не найден.].

The purpose of this paper is to simulate the DNS method of a horizontal heated pipe with the combined effect of TGC and longitudinal MF. Calculations for homogeneous heating have been performed. In the future, it's planned to consider one-sided heating in order to study the hypothesis of the occurrence of anomalous temperature pulsations with increasing Hartmann number in the longitudinal MF. This hypothesis is described in detail for a transverse MF [2].

* Corresponding author: akhmedagaev.r.m@gmail.com 


\section{Physical model}

The investigated geometry is shown in figure 1 . The length of the investigated section $(\mathrm{z})$ is assumed to be $5 \mathrm{~d}$. The radius of the computed area (r) varies from 0 to 1 .

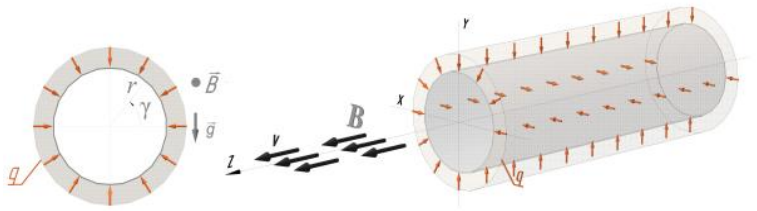

Fig. 1. The flow diagram.

The task is described by means of a system of equations, which includes equations of continuity - eq. (1), of motion - eq. (2) and of energy conservation - eq. (3). Such assumptions as constant properties, incompressible fluid are used, viscous dissipation is not taken into account, low-frequency approximation of electromagnetic hydrodynamics is applied. The final version of the system in a dimensionless form:

$$
\begin{gathered}
\nabla \cdot \boldsymbol{U}=0 \\
\frac{\partial \boldsymbol{U}}{\partial t}+(\boldsymbol{U} \cdot \nabla) \boldsymbol{U}=-\nabla p-\nabla \hat{p}+\frac{1}{R e} \cdot \nabla^{2} \boldsymbol{U}+\boldsymbol{F}_{b}+\boldsymbol{F}_{L} \\
\frac{\partial \theta}{\partial t}+(\boldsymbol{U} \cdot \nabla) \theta=\frac{1}{P e} \cdot \nabla^{2} \theta-U_{z} \cdot \frac{\mathrm{d} \bar{\theta}}{\mathrm{dZ}}
\end{gathered}
$$

The system of equations (1) - (3) is solved by the DNS method, the detailed algorithm of which is presented in [3].

\section{Results}

Today from experimental data [4] show that the longitudinal MF delays the transition from laminar flow regime to turbulent and it is observed in the growth of the critical Reynolds number, which increases with Hartmann number.

As an example, let us consider the dependence of the friction coefficient on the Reynolds number (fig. 2) and the effect of the longitudinal magnetic field on heat transfer (fig. 3). As the Hartmann number increases, a decrease in friction is observed due to suppression of turbulent velocity pulsations, which corresponds to experiments [4].

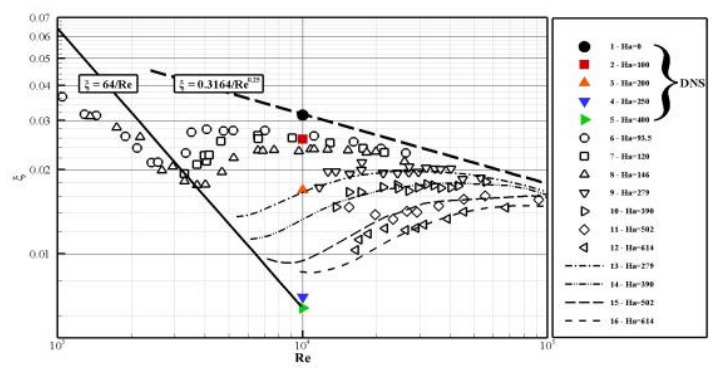

Fig. 2. The dependence of friction coefficient on Reynolds number, Ha=0-614: 1-5 - DNS for $\mathrm{Re}=10^{4} ; 6-12$ - experimental points [4]; 13-16 - interpolation lines [4].

A similar situation is observed for heat transfer, which decreases in a strong MF to a laminar Nusselt number. For Reynolds values, approximately equal to the critical Reynolds 
number for $H a \gg 1$ [5] - eq. (4), the difference between the DNS method and the experimental data increases. This is due to the fact that the hydrodynamic stabilization of the flow was not achieved in the experiments, in contrast to the calculation.

$$
R e_{c r}=30 \cdot H a
$$

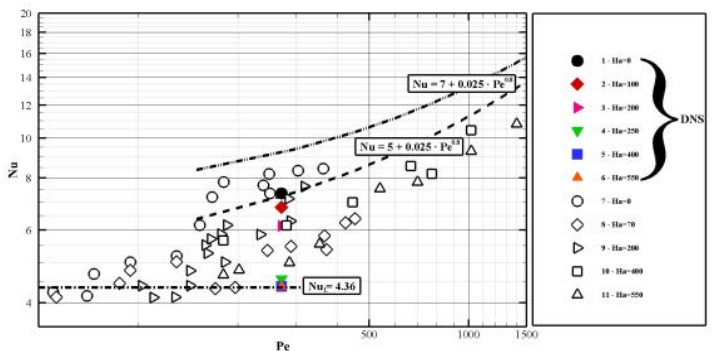

Fig. 3. The dependence of the Nusselt number from the Peclet number, $\mathrm{Ha}=0-550: 1-6-\mathrm{DNS}$ for $\mathrm{Pe}=272 ; 7-11-[4]$.

Now well-known is the effect of TGC on characteristics of hydrodynamics and heat and it depends on the orientation of the investigated area. In the joint conditions in strong MF effects of the TGC may be increase. A characteristic feature of the flow in these conditions is a substantial heterogeneity of the heat transfer coefficients and, therefore, wall temperature on the perimeter of the tube cross-section (fig. 4), which can lead to unacceptably high thermal stresses in the heat exchangers walls. The results of the DNS method are in good agreement with the experiment [6].

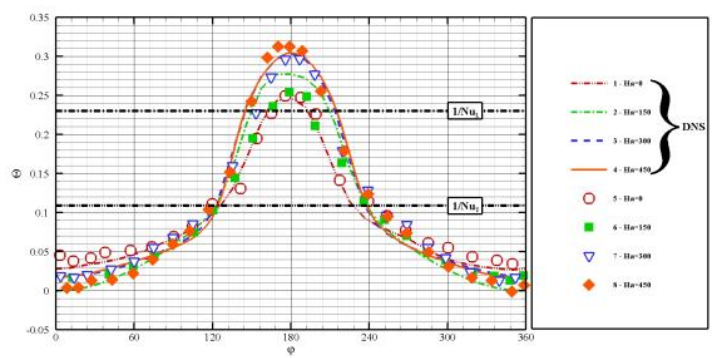

Fig. 4. Dimensionless wall temperature along the perimeter of the horizontal tube cross section, $\mathrm{Re}=10^{4}, \mathrm{Gr}=0.8 \cdot 10^{8}: 1-4-\mathrm{DNS} ; 5-8-[6]$.

In the horizontal tube affected by longitudinal MF TGC manifests itself in the form of large longitudinal vortices with axes parallel to the vector of the MF induction. MF stabilizes these vortices and, as a result, flow loses axial symmetry (fig. 5 for the temperature). Heat distribution becomes inhomogeneous in the tube cross-section and are formed of a zone of «degraded» heat transfer, which are determined by local Nusselt numbers less than the value for laminar mode $-\mathrm{Nu}_{\mathrm{L}}=4.36$.

For the averaged velocity fields (fig. 5 for the velocity), it is seen that TGC in strong MF influences more strongly than on the temperature fields: the maximum velocity is shifted to the lower part of the tube and stretched along the circumference. 
(a)

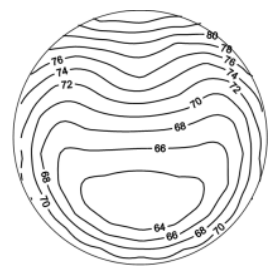

(b)

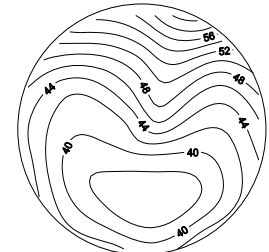

Experiment [6]
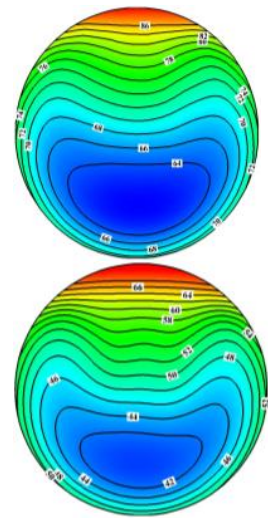

DNS

Temperature

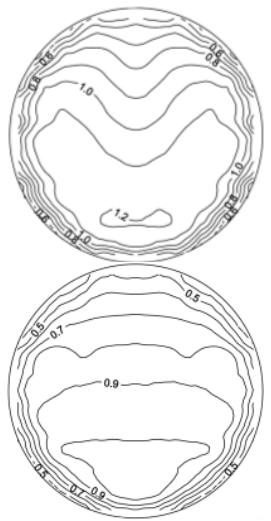

Experiment [6]

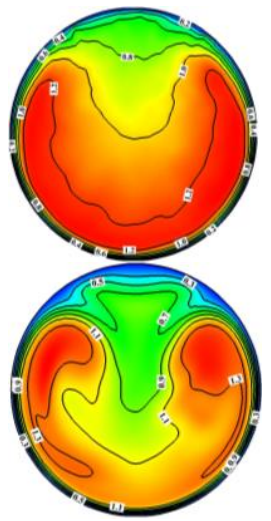

DNS

Velocity

Fig. 5. Fields of average temperature and longitudinal velocity component in the horizontal tube cross section, $\mathrm{Re}=10^{4}, \mathrm{Gr}=0.8 \cdot 10^{8}$ : a) $\mathrm{Ha}=0$; b) $\mathrm{Ha}=300$.

\section{Concluding remarks}

The authors confirmed the well-known fact that in the absence of TGC the longitudinal MF leads to suppression of turbulence with increasing Hartmann number.

In the case of homogeneous heating of the horizontal pipe wall with the combined influence of MF and TGC, the velocity field changes significantly and secondary convective currents appear. This leads to the appearance of inhomogeneity in the distribution of the local heat transfer coefficient along the perimeter of the wall and, as a consequence, the inhomogeneity of the temperature field. These effects can cause high thermal stresses, which should be taken into account when designing the ITER blanket.

The results were obtained during the implementation of the Draft State Order of the Ministry of Education and Science of the Russian Federation in the field of scientific activity No. 13.9619.2017 / 8.9

\section{References}

1. C.P.C. Wong, J.F. Salavy, Y. Kim, I. Kirillov, E. Rajendra Kumar, N.B. Morley, S. Tanaka, Y.C. Wu, Fusion Eng. Design 83, 850 (2008)

2. L.G. Genin, V.G. Zhilin, Y.P. Ivochkin, N.G. Razuvanov, I.A. Belyaev, Y.I. Listratov, V.G. Sviridov, Temperature fluctuations in a heated horizontal tube affected by transverse magnetic field, in: Proc. 8th PAMIR Conf. Fund. Appl. MHD, Borgo, Corsica, France, 37 (2011)

3. O. Zikanov, Y. Listratov, V.G. Sviridov, J. Fluid Mech. 720486 (2013)

4. L.G. Genin, V.G. Sviridov, Hydrodynamics and heat transfer of MHD flows in channels (Moscow, MPEI Publ., 2001) [in Russian]

5. F.W. Fraim, W.H. Heiser, J. Fluid Mech. 33, 2 (1968)

6. I.A. Belyaev, L.G. Genin, Ya.I. Listratov, I.A. Melnikov, V.G. Sviridov, E.V. Sviridov, Yu.P. Ivochkin, N.G. Razuvanov, Yu.S. Shpansky, Megnetohydrodynamics 49, 1 (2013) 